\title{
Universiteit
}

Leiden

The Netherlands

\section{Force and weight distributions in granular media: Effects of contact geometry}

Snoeijer, J.H.; Hecke, M.L. van; Somfai, E.; Saarloos, W. van

\section{Citation}

Snoeijer, J. H., Hecke, M. L. van, Somfai, E., \& Saarloos, W. van. (2003). Force and weight distributions in granular media: Effects of contact geometry. Physical Review E, 67(3), 030302. doi:10.1103/PhysRevE.67.030302

Version: $\quad$ Publisher's Version

License: $\quad$ Leiden University Non-exclusive license

Downloaded from: https://hdl.handle.net/1887/66544

Note: To cite this publication please use the final published version (if applicable). 


\title{
Force and weight distributions in granular media: Effects of contact geometry
}

\author{
Jacco H. Snoeijer, ${ }^{1}$ Martin van Hecke, ${ }^{2}$ Ellák Somfai, ${ }^{1, *}$ and Wim van Saarloos ${ }^{1}$ \\ ${ }^{1}$ Instituut-Lorentz, Universiteit Leiden, Postbus 9506, 2300 RA Leiden, The Netherlands \\ ${ }^{2}$ Kamerlingh Onnes Lab, Universiteit Leiden, Postbus 9504, 2300 RA Leiden, The Netherlands
}

(Received 24 October 2002; published 25 March 2003)

\begin{abstract}
The influence of the local contact network on interparticle forces and effective particle weights is studied in simulations of two-dimensional packings of frictionless, Hertzian spheres. The weight distribution $\mathcal{P}(w)$ changes qualitatively when approaching a boundary and differs for regular and irregular packings, while the interparticle force distribution $P(f)$ is robust. We provide examples where $\mathcal{P}(w)$ at the boundary, which is the quantity probed experimentally, deviates substantially from $P(f)$ in the bulk. Discrepancies between the $\mathcal{P}(w)$ 's predicted by the $q$ model and measured in experiments are due to differences in the contact geometry.
\end{abstract}

DOI: 10.1103/PhysRevE.67.030302

PACS number(s): 45.70.Cc, 05.40.-a, 46.65.+g

A crucial property of granular materials is their heterogeneity [1]. In particular, the strong fluctuations of interparticle forces and the organization of the largest of these in tenuous force networks have recently attracted considerable attention [2-9]. The most basic characterization of the force network is the probability distribution of the contact forces. Measurements [2-6], numerical simulations [7,8], and theory [9] all find that force distributions decay exponentially for large force [10], albeit with nonuniversal exponents. The behavior for small forces is less well settled but relevant: it may indicate arching [11] or jamming [12].

In this paper, we will unravel the effect of the local contact geometry on the distributions of interparticle force $F$ and effective particle weight $W$; the weight is defined as the sum of the vertical components of all downward pointing forces on a particle [see Fig. 1(b)]. While the distribution of forces $F$ is the primary object one ultimately wishes to characterize, it is difficult to access experimentally. In experiments where particle-wall forces are measured by means of imprints on carbon paper [2-4] or force sensors [5] one probes the weight of the bottom particles. The weight is also the prime quantity described by the $q$ model, which is a lattice model in which weights are randomly redistributed over a fixed number of supporting grains [9].

Our central point is that while the distribution of $F$ is robust, the distribution of $W$ is profoundly influenced by the contact geometry, in particular by the number of downward pointing contact forces $n_{c}$. This has the following consequences.

(i) The bulk and bottom weight distributions are qualitatively different due to geometrical effects: on an average $n_{c}$ is smaller for bottom than for bulk particles [see Fig. 1(a)].

(ii) For small weights, the weight distribution of the $q$ model is qualitatively different from distributions observed experimentally; this is due to the fixed contact geometry (fixed $n_{c}$ ) of the $q$ model.

(iii) The distributions of bottom weights and bulk forces are in general different; regular contact geometries or highly compressed packings [4] exhibit substantial differences be-

\footnotetext{
*Present address: Department of Physics, University of Warwick, Coventry CV4 7AL, UK.
}

tween bulk force and bottom weight distributions. For packings in which many bottom particles have $n_{c}=1$, however, the bulk force and bottom weight distributions are similar.

We will focus on frictionless, Hertzian spheres under gravity. The particle-boundary forces are then purely vertical, and we will refer to their magnitude as the effective weight $W$ of a particle. To allow comparison to the $q$ model, we define the effective weight also for bulk particles [see Fig. 1(b)]:

$$
W_{j} \equiv m_{j} g+\sum_{\langle i\rangle}\left(\vec{F}_{i j}\right)_{z}
$$

Here $m_{j}$ denotes mass, $g$ denotes gravity, $\vec{F}_{i j}$ are the interparticle forces, and the sum runs over $n_{c}$ "up" contacts, associated with the particles that exert a force on particle $j$ from above. In the following, we rescale $W$ and $F$ to their average values at a certain height; we write the rescaled weights and forces as $w$ and $f$ with distributions $\mathcal{P}(w)$ and $P(f)$.

We will probe $\mathcal{P}(w)$ and $P(f)$ by examining boundary effects in an irregular packing (Figs. 2 and 3), and by studying a regular packing where the packing order can be broken by curving the boundary (Fig. 4). Our framework can now be summarized as follows. The geometry of the contact network has a strong effect on $\mathcal{P}(w)$, but $P(f)$ is very robust. The weight distribution for particles with a given $n_{c}, \mathcal{P}_{n_{c}}(w)$, is robust and behaves as $w^{n_{c}-1}$ for small $w . \mathcal{P}(w)$ can be de-
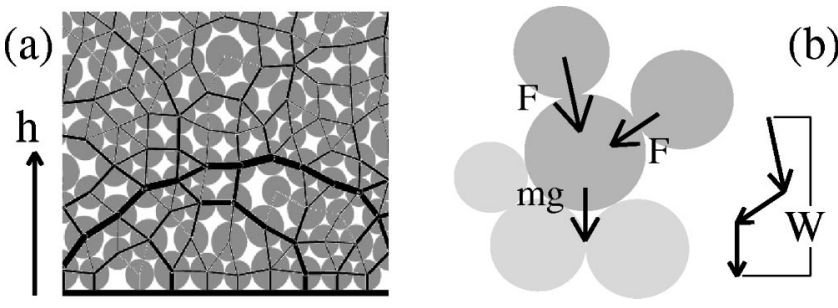

FIG. 1. (a) Detail of a typical packing in our simulations; the height $h$ denotes the distance from the bottom. The force network is represented by the black lines whose thickness represents the force magnitude. (b) Definition of interparticle forces $F$ and weight $W$ for a particle with $n_{c}=2$. 

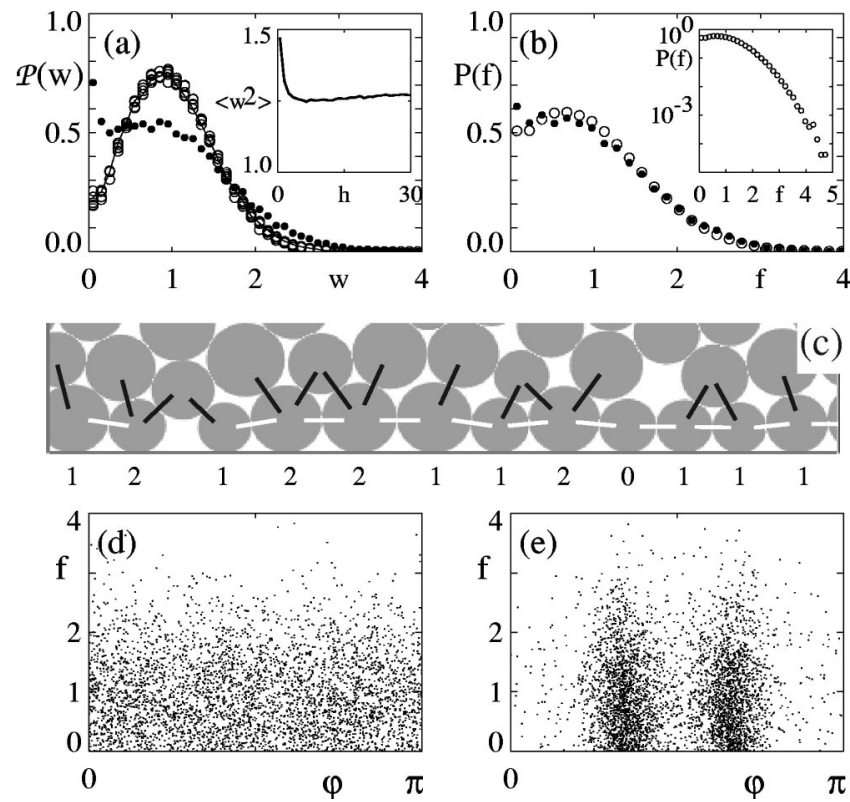

FIG. 2. (a) The weight distribution $\mathcal{P}(w)$ at various heights between 10 and 30 in the bulk (open circles) for $2<h<3$ (full curve) and at the bottom (dots). Inset: $\left\langle w^{2}\right\rangle$ which quantifies the width of $\mathcal{P}(w)$ as a function of height $h$. The sharp transition of $\mathcal{P}(w)$ near the bottom is clearly visible. (b) $P(f)$ in the bulk (open circles) and for the layer-to-layer forces near the bottom (dots); the inset shows $P(f)$ for bulk forces on a log-lin scale. (c) Detail of a typical packing showing dominance near the bottom of layer-tolayer forces (black lines) to intralayer forces (white lines) in determining $w$. The numbers show the values of $n_{c}$ for the respective bottom particles. (d),(e) Scatter plot of $\left(f_{i j}, \varphi_{i j}\right)$ for (d) the bulk forces and (e) the layer-to-layer forces near the bottom.
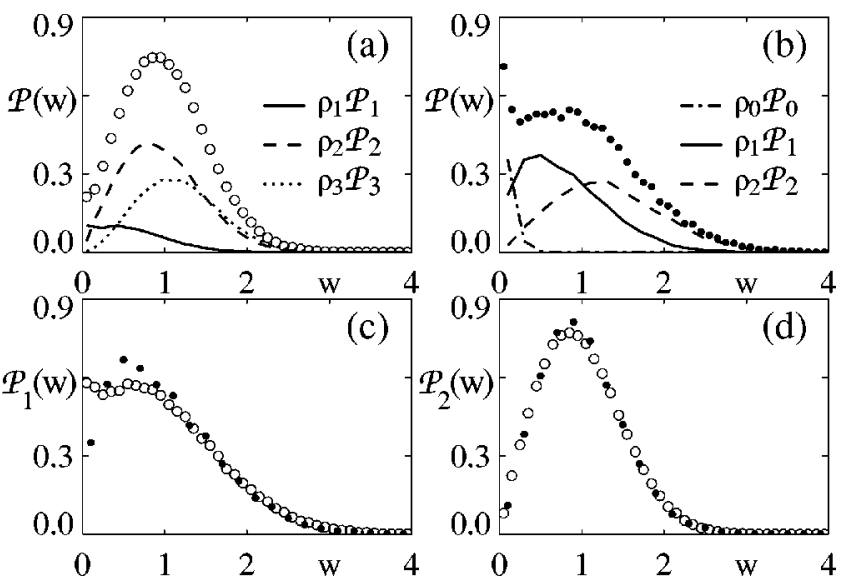

FIG. 3. (a,b) Decomposition of $\mathcal{P}(w)$ according to Eq. (3) (a) in the bulk (open circles) and (b) at the bottom (dots); the measured bulk values for the fractions $\left\{\rho_{0}, \rho_{1}, \rho_{2}, \rho_{3}\right\}$ in Eq. (3) are $\{0.01$, $0.11,0.52,0.36\}$ and the bottom values are $\{0.08,0.46,0.44,0.02\}$; as explained after Eq. (3), we excluded the intralayer, almost horizontal forces at the bottom. (c),(d) When rescaled to the average value for each distribution function, $\mathcal{P}_{1}(w)(\mathrm{c})$ and $\mathcal{P}_{2}(w)$ (d) are essentially the same in the bulk (open circles) and at the bottom (dots).
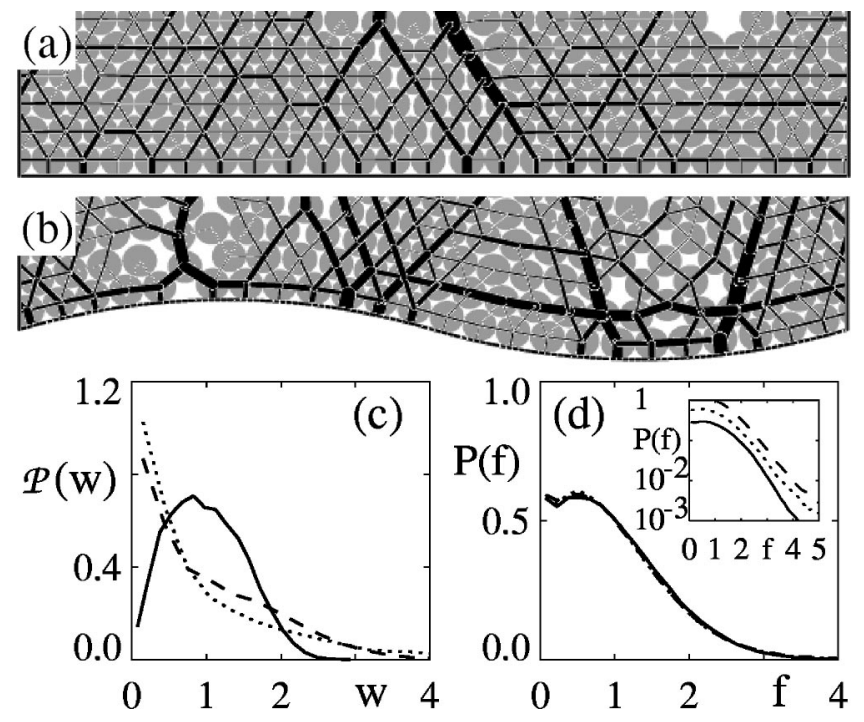

FIG. 4. (a),(b) Packing and force networks in a weakly polydisperse packing near a flat bottom (a) and a curved bottom (two circle segments of radius 20 glued together) (b). (c) Distribution of weights $\mathcal{P}(w)$ on the flat bottom (solid line), convex curved bottom (dashed line), and the concave curved bottom (dotted line). The various shapes originate from the corresponding $\left\{\rho_{0}, \rho_{1}, \rho_{2}\right\}:\{0.00$, $0.10,0.90\},\{0.02,0.39,0.58\}$, and $\{0.04,0.46,0.50\}$, respectively. (d) $P(f)$ in the bulk ( $h$ ranging from 10 to 20 ) for the flat, convex, and concave bottoms; the inset shows the $P(f)$ 's on a log-lin scale (the curves are offset for clarity).

composed as $\mathcal{P}(w)=\sum_{n_{c}} \rho_{n_{c}} \mathcal{P}_{n_{c}}(w)$, where $\rho_{n_{c}}$ are the fractions of particles that have $n_{c}=0,1,2, \ldots$ "up" contacts. Differences of $\rho_{n_{c}}$ between boundary particles and bulk particles, and between irregular and regular ( $q$ model-like) packings explain the different $\mathcal{P}(w)$ 's for these cases. When $\rho_{0}$ and $\rho_{1}$ are large, the total weight distributions $\mathcal{P}(w)$ exhibits a plateau at small weights and a slow decay at large weights; when $\rho_{2}$ and $\rho_{3}$ become large, $\mathcal{P}(w)$ becomes sharply peaked. In this way, $\mathcal{P}(w)$ 's small weight behavior as well as its exponential decay rate for large weights [10] reflect the packing geometry.

(a) Numerical method. Our two-dimensional (2D) packings consist of frictionless spheres (3D) under gravity; the particles interact through normal Hertzian forces, where $f \propto d^{3 / 2}$ and $d$ denotes the overlap distance [13]. Unless noted otherwise, the material constants and gravity are chosen such that a particle deforms $0.1 \%$ under its own weight, and the particle radii are drawn from a flat distribution between $0.4<r<0.6$. Masses are proportional to the cube of the radii. The container has a width of 24 , employs periodic boundary conditions in the horizontal direction, and has a bottom consisting of a fixed hard support. The data shown in this paper were obtained from 1100 realizations of 1180 particles each. We construct our packings by letting the particles relax from a gaslike state.

(b) Boundary effects. In Figs. 2(a) and 2(b), the distributions for the weight and interparticle forces are shown for bottom (dots) and bulk (open circles) particles. For $\mathcal{P}(w)$ we observe a substantial difference between bulk and bottom 
particles, and the transition between the bulk and bottom behaviors is remarkably sharp: in the slice $2<h<3$ (full curve), the weight distribution is already bulklike [Fig. 2(a) and inset]. The force distribution $P(f)$ is more robust; the only difference between bulk and bottom distributions is the change of the small peak around $f=0.7$ for bulk forces to a plateau for $P(f)$ near the bottom [Fig. 2(b)]. It is intriguing to note that this change is reminiscent of what is proposed as an identification of the jamming transition [12]. The good agreement between $P(f)_{\text {bulk }}$ and $\mathcal{P}_{\text {bottom }}(w)$ for $w \gtrsim 0.3$ is fortuitous and, as we will show below, due to the large fraction of bottom particles that have $n_{c}=0$ or 1 .

Figure 2(c) illustrates that due to particular geometry of a packing near the bottom, the interparticle forces naturally divide up into almost horizontal intralayer forces and "layerto-layer" forces. The distributions of the contact angles $\varphi_{i j}$ are shown in Figs. 2(d) and 2(e) for bulk and boundary contacts. In the bulk the contact angles are uniformly distributed and independent of $|\vec{f}|$ : our packing is isotropic. Near the boundary, however, this isotropy is strongly broken: the angles of the "layer-to-layer" forces between bottom particles and those in the layer above are concentrated around $\pi / 3$ and $2 \pi / 3$.

Decomposition of $\mathcal{P}(w)$. To understand the change of $\mathcal{P}(w)$ near the bottom, consider the typical packing of Fig. 2(c). The forces between neighboring bottom particles are almost purely horizontal, so these hardly contribute to the total force on the particle from above [the "weight" in Eq. (1)]. Thus, the average value of $n_{c}$, the number of particles that contribute to its weight, is on an average lower at the bottom than in the bulk. Let us now argue why the probability of finding a small value of $w$ increases with smaller $n_{c}$. Consider Eq. (1) for fixed $n_{c}$ and analyze $\mathcal{P}_{n_{c}}(w)$, the weight probabilities restricted to particles of given $n_{c}$. As long as the joint probability distribution of the interparticle forces remains finite for small forces, it follows from a phase-space argument that

$$
\mathcal{P}_{n_{c}}(w) \propto w^{n_{c}-1} \quad \text { for } \quad w \rightarrow 0,
$$

for all $n_{c} \geqslant 1$ [14]. The particles that do not feel a force from above have $n_{c}=0$ and give a $\delta$-like contribution at $W$ $=m g$; for deep layers this occurs for $w \ll 1$.

To check the validity of this idea, we have determined $\mathcal{P}_{n_{c}}(w)$ both in the bulk and near the bottom by determining $n_{c}$ for each particle and decomposing the weight distribution $\mathcal{P}(w)$ into the $\mathcal{P}_{n_{c}}(w)$ 's,

$$
\mathcal{P}(w)=\sum_{n_{c}} \rho_{n_{c}} \mathcal{P}_{n_{c}}(w) .
$$

In Figs. 3(a) and 3(b) we show this decomposition for the bulk and bottom $\mathcal{P}(w)$ 's, while $\mathcal{P}_{1}(w)$ and $\mathcal{P}_{2}(w)$ are shown in Figs. 3(c) and 3(d). In determining the value of $n_{c}$ we explicitly exclude the intralayer bottom contacts; for the strongly polydisperse packings, these correspond to angles that deviate less than $13^{\circ}$ from the horizontal. For our estimates of the bulk $\rho_{n_{c}}$ 's we do not exclude any forces, i.e., the "cutoff angle" is strictly $0^{\circ}$. Since the bulk is isotropic, a cutoff angle of $13^{\circ}$ leads to a change of the order of $15 \%$, far too little to explain the change of $\rho_{1}$ by a factor of more than 4. For simplicity, we therefore have kept a cutoff angle of $0^{\circ}$ in the bulk.

The crucial difference between bottom and bulk are the fractions $\rho_{n_{c}}$ of particles that experience $n_{c}$ other particles pressing on them from above; the $\mathcal{P}_{n_{c}}(w)$ 's obey Eq. (2) and are very similar, except for a small difference between the bulk and bottom $\mathcal{P}_{1}(w)$ 's for small $w$ [15].

The behavior of $\mathcal{P}(w)$ for small $w$ is dominated by $\rho_{0}$ and $\rho_{1}$, but these fractions also affect the steepness of the tail for $\mathcal{P}(w)$ via the normalization conditions $\int d w \mathcal{P}(w)=1$ and $\int d w w \mathcal{P}(w)=1$. A small value of $\lim _{w \downarrow 0} \mathcal{P}(w)$ leads to a sharply peaked distribution that falls off with a large exponent [Fig. 2(a) for the bulk and Fig. 3(d)], while a plateau at small $w$ leads to a slower exponential decay at large $w$ [Fig. 2(a) for the bottom and Fig. 3(c]. The contact geometry affects the small weight distribution and hence, via the normalization constraints, the exponential decay rate of the largeweight tail [10].

(c) Regular packings. We have seen above that the weight distribution $\mathcal{P}(w)$ is very sensitive to the local packing geometry, while the distribution of interparticle forces is robust. For the particular example shown in Figs. 2 and 3, however, $P_{\text {bulk }}(f)$ and $\mathcal{P}_{\text {bottom }}(w)$ look rather similar due to a large amount of particles with $n_{c}=1$. In order to provide an example where $P(f)$ and $\mathcal{P}(w)$ are radically different, and to test the robustness of $P(f)$, we consider a packing of weakly polydisperse particles, $0.49<r<0.51$. Above a flat bottom, the particles form an almost perfect hexagonal packing [Fig. 4(a)], while for a curved bottom this order is broken [Fig. 4(b)]. The flat bottom leads to particles with mostly $n_{c}=2$, and indeed $\mathcal{P}(w) \sim w$ for small $w$ in agreement with Eq. (2) [see Fig. 4(c)]. For the curved bottom a dramatic change in the fractions $\rho_{n_{c}}$ and correspondingly $\mathcal{P}(w)$ occurs [Fig. 4(c)]. $P(f)$ in the bulk are all indistinguishable [see Fig. 4(d)] and very similar to $P(f)$ in the strongly polydisperse case [Fig. 2(b)]: even major changes in the contact network do not affect the bulk $P(f)$.

In experiments, the differences between regular and irregular packings were found to be minute [3]. However, the experimental data for regular packings shows evidence for a $\delta$ peak at $w=0$, which indicates that there are $n_{c}=0$, and likely also $n_{c}=1$ particles present. Even though the experimental packing is regular, the contact network is apparently not, possibly due to friction and the hardness of the particles.

The regular packing also clarifies the discrepancy between the $\mathcal{P}(w)$ 's in measurements and $q$ model [9]. In such a lattice model, the weight of a particle is randomly distributed over $n_{c}$ neighbors. In the simplest case $n_{c}=2$; the resulting $\mathcal{P}(w)$ is then qualitatively similar to what we found for the flat bottom; in particular, $\mathcal{P}(w) \sim w$ for small $w$ [9]. This is due to the absence of particles with $n_{c}=1$ : in simulations of the $q$ model with random connectivities, we found a variety of $\mathcal{P}(w)$ 's with finite $\lim _{w \downarrow 0} \mathcal{P}(w)$ once $\rho_{1}>0$ [16]. 
Finally, note that $f_{q} \equiv q w$ in the $q$ model is more like a contact force, and that $P\left(f_{q}\right)$ remains finite for small $f_{q}[9,16]$.

(d) Perspective. Our message is clear: in experiments in which the forces on a boundary are probed, one measures effectively the weight distribution $\mathcal{P}(w)$ that is sensitive to the local packing near the bottom and which a priori is different from the robust force distribution $P(f)$. Why $P(f)$ is so robust remains an intriguing open issue.

From the experimentally obtained $\mathcal{P}(w)$, one can estimate the fractions $\rho_{0}$ and $\rho_{1}$ which dominate the small weight distribution. The height of the peak at $w=0$, clearly present in Fig. 5 of Ref. [3] and in Ref. [4] is given by $\rho_{0} / \Delta w$, where $\Delta w$ is the bin width. Ignoring this peak and estimating $\lim _{w \downarrow 0} \mathcal{P}(w)$ gives the value of $\rho_{1} P_{1}(0)$; direct measurements of $\rho_{1}$ would yield $P_{1}(0)$. Based on these considerations, it seems that for most experimentally observed packings, $\rho_{0}$ and $\rho_{1}$ are substantial at the boundary, so that $\mathcal{P}_{\text {bottom }}(w)$ is similar to $P(f)_{\text {bulk }}$ (apart from a $\delta$ peak at $w$ $=0)$. The same argument probably holds for recent simulations by Silbert et al. [17].

However, in recent experimental data on highly deformed packings of rubber beads [4], a clear decrease in the $\delta$ peak and $\lim _{w \downarrow 0} \mathcal{P}(w)$ is visible. We think that these trends are entirely consistent with a decrease of $\rho_{0}$ and $\rho_{1}$ and increase of $\rho_{2}$ and $\rho_{3}$, as expected for highly deformed packings. Such changes in the $\rho$ 's would also lead to a steepening of the exponential tail of $\mathcal{P}(w)$; this steepening is clearly visible in the data of Ref. [4].

To further test our framework in experiments, direct measurements of the fractions $\rho_{i}$ would be very welcome. Alternatively, one can directly influence these fractions by placing a layer of relatively small or large beads at the bottom. For small beads, $\rho_{0}$ and $\rho_{1}$ will be enhanced, leading to a large $\mathcal{P}(w)$ for small $w$, and a slow exponential decay for large $w$. Relatively large bottom beads will suppress $\rho_{0}$ and $\rho_{1}$ and increase $\rho_{2}$, etc.; the $\lim _{w \downarrow 0} \mathcal{P}(w)$ is then suppressed, the exponential decay becomes steep and $\mathcal{P}(w)$ appears strongly peaked. Since $P(f)$ in the bulk is not influenced by a single layer of boundary grains, this may be the simplest way to test our framework.

An important issue for future study is clearly the role of friction and dimensionality. Our numerical study has been done in two dimensions with frictionless spheres; however, recent studies indicate [8] that the coordination number for $3 \mathrm{D}$ packings with friction is similar to those of $2 \mathrm{D}$ frictionless packings. Qualitatively, the picture with which we have advanced is therefore expected to capture the realistic case of three dimensions with friction because our phase-space arguments are independent of dimension.

We are grateful to Martin Howard and Hans van Leeuwen for numerous illuminating discussions. J.H.S. and E.S. acknowledge support from the FOM foundation.
[1] H.M. Jaeger, S.R. Nagel, and R.P. Behringer, Rev. Mod. Phys. 68, 1259 (1996); P.G. de Gennes, ibid. 71, 374 (1999); L.P. Kadanoff, ibid. 71, 435 (1999).

[2] D.M. Mueth, H.M. Jaeger, and S.R. Nagel, Phys. Rev. E 57, 3164 (1998).

[3] D.L. Blair et al., Phys. Rev. E 63, 041304 (2001).

[4] J.M. Erikson et al., Phys. Rev. E 66, 040301 (2002).

[5] G. Løvoll, K.J. Måløy, and E.G. Flekkøy, Phys. Rev. E 60, 5872 (1999).

[6] D. Howell, R.P. Behringer, and C. Veje, Phys. Rev. Lett. 82, 5241 (1999).

[7] F. Radjai et al., Phys. Rev. Lett. 77, 274 (1996); S. Luding, Phys. Rev. E 55, 4720 (1997); F. Radjai et al., Phys. Rev. Lett. 80, 61 (1998); A.V. Tkachenko and T.A. Witten, Phys. Rev. E 62, 2510 (2000); S.J. Antony, ibid. 63, 011302 (2000); C.S. O’Hern et al., Phys. Rev. Lett. 88, 075507 (2002).

[8] H.A. Makse, D.L. Johnson, and L.M. Schwartz, Phys. Rev. Lett. 84, 4160 (2000).

[9] C. Liu et al., Science 269, 513 (1995); S.N. Coppersmith et al., Phys. Rev. E 53, 4673 (1996).
[10] This tail is only exponential if the particle deformations are sufficiently small [8]. Our maximal deformations are $2 \%$ and our tails are not perfectly exponential.

[11] P. Claudin et al., Phys. Rev. E 57, 4441 (1998).

[12] A.J. Liu and S.R. Nagel, Nature (London) 396, 21 (1998); C.S. O'Hern et al., Phys. Rev. Lett. 86, 111 (2001); I.K. Ono et al., ibid. 89, 095703 (2002).

[13] Contact Mechanics, edited by K.L. Johnson (Cambridge University Press, Cambridge, England 1985).

[14] Such scaling is also implicit in the $q$ model, although there $n_{c} \geqslant 2$ so that $\mathcal{P}(0)=0$.

[15] Bottom particles with $n_{c}=0$ or 1 are typically smaller than average [see Fig. 2(c)]. For these particles, the intralayer "almost horizontal" forces do contribute slightly to the weights, which enhances the probability for small (but nonzero) weights at the expense of $\mathcal{P}(0)$; this effect is visible for $\mathcal{P}_{0, \text { bottom }}(w)$ and $\mathcal{P}_{1, \text { bottom }}(w)$ in Fig. 3(b).

[16] J.H. Snoeijer et al. (unpublished).

[17] L.E. Silbert, G.S. Grest, and J.W. Landry, Phys. Rev. E 66, 061303 (2002). 